\title{
Nonlinear Shear Rheology of Entangled Polymer
}

\section{Rings:}

\section{Supplementary Information}

\author{
Daniele Parisi, ${ }^{\dagger, \ddagger}$ Salvatore Costanzo, ${ }^{\dagger, \uparrow}$ Youncheol Jeong, ${ }^{\S}$ Junyoung Ahn, ${ }^{\S}$ \\ Taihyun Chang, ${ }^{\S}$ Dimitris Vlassopoulos, ${ }^{, \dagger}$ Jonathan D. Halverson," Kurt \\ Kremer, ${ }^{\perp}$ Ting Ge, ${ }^{\#}$ Michael Rubinstein,, ${ }^{* @}$ Gary S. Grest, ${ }^{\triangle}$ Watee Srinin, ${ }^{\nabla}$ \\ and Alexander Y. Grosberg ${ }^{*, \dagger \dagger}$ \\ $\dagger$ FORTH and University of Crete, Greece \\ $\ddagger$ Penn State University, USA \\ ФUniversity of Naples, Italy \\ $\S$ Pohang University of Science and Technology, South Korea \\ ||Princeton University, USA \\ $\perp$ Max Planck Institute for Polymer Research, Germany \\ \#University of South Carolina, USA \\ @ Duke University, USA and Hokkaido University, Japan \\ $\triangle$ Sandia National Laboratory, USA \\ $\nabla$ Naresuan University, Thailand \\ $\dagger \dagger$ New York University, USA
}

E-mail: dvlasso@iesl.forth.gr; michael.rubinstein@duke.edu; ayg1@nyu.edu 


\section{Table of Content for Supplementary Information}

1. Experimental results $\quad$ page S2

(a) Rings characterization: Materials $\quad$ page S2

(b) Viscosity measurements page S3

$\begin{array}{ll}\text { (c) Viscosity data } & \text { page } \mathrm{S} 4\end{array}$

2. Simulation results page S5

3. Shear slit model page S10

(a) Analytical expressions and limits page S10

(b) Interpolation expressions for relaxation modulus $G(t) \quad$ page S11

- Rings

page $\mathrm{S} 11$

- Linear chains

page S13

- The limit of infinite rings

page $\mathrm{S} 14$

$\begin{array}{ll}\text { 4. Cox-Merz rule } & \text { page S17 }\end{array}$

$\begin{array}{ll}\text { (a) Two forms of Cox-Merz rule } & \text { page S17 }\end{array}$

(b) Two forms of Cox-Merz rule and experiments $\quad$ page S17

(c) Two forms of Cox-Merz rule and simulations $\quad$ page S18

(d) Mathematics of two forms of Cox-Merz rule page S19

5. Ring Stretched along a Line page S21

\section{Experimental Methods and Results}

\section{1a. Rings characterization: Materials}

The LCCC technique can effectively purify the ring samples, making the residual linear contamination hardly detectable by any analysis including SEC, LCCC and MALDI-MS. We 
emphasize that obtaining true critical conditions, where linear polymers of different molar masses are eluted at the same time, is important. We determined the critical condition unambiguously (co-elution condition of different linear samples) by changing the mobile phase composition and/or column temperature. The SEC chromatograms in Figure S1a shows the comparison between the elution curves before and after LCCC fractionation. The results clearly indicate a very effective purification of the ring polymer from the residual linear precursor. Figure S1b shows the comparison between the chromatograms of the fractions of ring polymer and its linear precursor that had been separated by LCCC. A stable and clean (high $S / N$ ratio) baseline is very important, and here we can see that the LCCC separations were performed unambiguously down to the baseline. The elution peaks of the ring samples correspond to larger values of the elution volume with respect to the linear samples. This corroborates the known fact that, due to their molecular structure, ring polymers have smaller size compared to linear polymers of the same $M_{w}$. The weight-average molar mass, $M_{w}$, and the polydispersity, $M_{w} / M_{n}$, of the LCCC-purified ring polymers (coded as PS-84k ring and PS-185k ring, respectively) determined by SEC-light scattering detection were 84 $\mathrm{kg} / \mathrm{mol}$ and $185 \mathrm{~kg} / \mathrm{mol}$ and lower than 1.01, respectively.

\section{1b. Viscosity measurements}

Rheological measurements were performed on a strain-controlled ARES rheometer (TA Instruments, USA), equipped with a force rebalance transducer (2KFRTN1). In order to avoid artifacts due to edge fracture, a home-made cone partitioned-plate (CPP) fixture was used. Details can be found in references, ${ }^{27,50}$ and more complete discussion in ref. ${ }^{51}$

Briefly, the bottom tool is a standard stainless-steel cone with a diameter of $25 \mathrm{~mm}$, an angle of $0.1 \mathrm{rad}$, and a nominal truncation of $51 \mu \mathrm{m}$. The upper tool is a partitioned plate, divided into an inner $6 \mathrm{~mm}$ plate and an outer corona. The latter has an inner diameter ranging from $6.16 \mathrm{~mm}$ to $6.20 \mathrm{~mm}$ (for different plates used) and an outer diameter of $20 \mathrm{~mm}$. The inner plate is the measuring part of the geometry while the outer non-measuring corona 

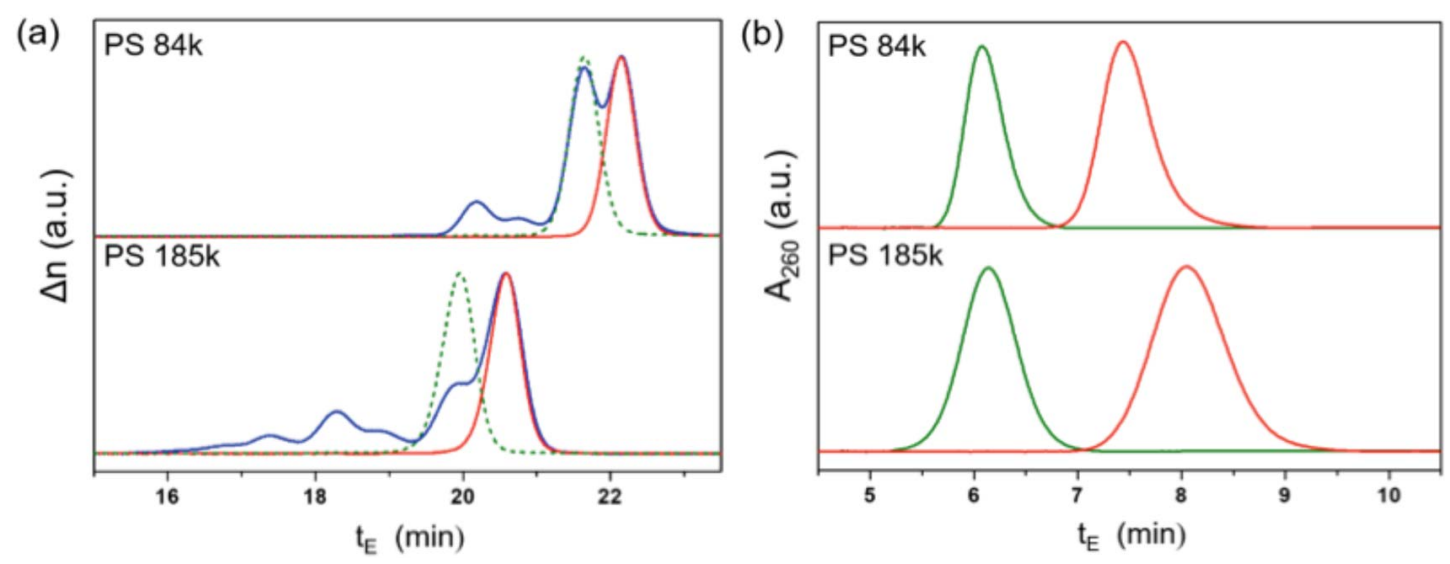

Figure S1: (a) SEC chromatograms of two PS precursors (green dashed lines), corresponding cyclization products (blue solid lines) and PS ring products after LCCC fractionation (red solid lines). Separation condition: Three mixed bed columns; Eluent: THF at a flow rate of $0.7 \mathrm{~mL} / \mathrm{min}$; Column temperature: $40.0^{\circ} \mathrm{C}$. (b) LCCC chromatograms of two PS linear precursors (green solid lines) and purified PS ring products (red solid lines). Separation condition: Nucleosil C18, $250 \times 4.6 \mathrm{~mm}, 300 \AA$, $5 \mu \mathrm{m}$. Eluent: THF/n-hexane $(57 / 43, \mathrm{v} / \mathrm{v})$ at a flow rate of $0.5 \mathrm{~mL} / \mathrm{min}$. Column temperature: $37.0^{\circ} \mathrm{C}$.

serves to extend the upper plate, in order to house larger samples with respect to the effective measured volumes. In strong shear flows of viscoelastic materials, a fracture arises at the rim of the sample, and propagates inwards during shearing. However, with the use of the CPP reliable viscosity measurements are possible until the fracture reaches the measured inner volume. The inner plate is directly attached to the transducer, while the outer corona is attached to the head of the rheometer by means of a hollow bridge equipped with vertical and horizontal homemade translation stages for the alignment of the two concentric partitions. Once the geometry is aligned, it can be fixed by means of horizontal and vertical screws. The entire fixture is housed into the standard convection oven of the ARES rheometer (with nitrogen gas flow to reduce the risk of degradation), hence ensuring excellent temperature control with a resolution of $\pm 0.1^{\circ} \mathrm{C}$.

\section{1c. Viscosity data}

Table S1 presents numerical values of terminal relaxation times $\tau_{\text {relax }}$ and zero shear rate viscosities $\eta_{0}$ for all experimentally tested samples. While determination of $\tau_{\text {relax }}$ in oscillatory 
experiment was described in the main text (see Figure 3 and text around it), here we comment on determination of $\eta_{0}$. We used several different methods and established that they are all consistent to within a few percent. In one method, we simply used the result of the steady shear measurement $\eta(\dot{\gamma})$ at the smallest accessible shear rate; as it is seen in the Table S1, this smallest $\dot{\gamma}$ corresponds to small Wi, which justifies the use of $\eta(\dot{\gamma})$ at this $\dot{\gamma}$ as a proxy for $\eta_{0}$. Our second method is based on the oscillatory experiment, where we performed dynamic measurements and then used $\left|\eta^{*}(\omega)\right|$ at the smallest accessible frequency, corresponding to small De; note that for the 185k linear and ring samples, the respective values extracted from long-time creep measurements (Fig. 3) are identical to those reported in Table S1 within 1\%). The third method employs time dependent stress relaxation modulus, which we determined numerically from the dynamic oscillatory shear data, and then we found $\eta_{0}$ via numerical integration $\eta_{0}=\int_{0}^{\infty} G(t) d t=\int_{0}^{\infty} t G(t) d \ln t$. In fact, we used two more methods (that we do not show), namely, we used either $\eta(\dot{\gamma})$ or $\left|\eta^{*}(\omega)\right|$ data and fitted them to Carreau-Yasuda equation (1) using previously determined values of $\alpha$ for each sample (Fig. 5) and considering $\eta_{0}$ as an adjustable parameter. These methods also yielded nearly identical results.

Table S2 lists result for experimental steady state viscosity.

Table S1: Terminal relaxation times and zero shear rate viscosities for all samples. We used this data to normalize both axes to transform Figure 4 into Figure 5. The last three columns show good agreement between zero shear rate viscosity $\eta_{0}$ values obtained by taking the steady state viscosity $\eta(\dot{\gamma})$ at the smallest shear rate $\dot{\gamma}$ (corresponding to sufficiently small Weissenberg numbers), by taking the absolute value of complex viscosity $\left|\eta^{*}(\omega)\right|$ at the smallest frequency (corresponding to sufficiently small Deborah numbers), or by integrating the shear stress relaxation modulus.

\begin{tabular}{|c|c|c|c|c|c|c|}
\hline & $\begin{array}{c}\tau_{\text {relax }} \\
{[\mathrm{s}]}\end{array}$ & $\begin{array}{c}\eta_{0} \text { from } \\
\eta(\dot{\gamma}) \\
{\left[\begin{array}{ll}\mathrm{kPa} & \mathrm{s}\end{array}\right]}\end{array}$ & $\begin{array}{l}\text { allest } \dot{\gamma} \\
\mathrm{Wi}\end{array}$ & $\begin{array}{c}\eta_{0} \text { from } \\
\left|\eta^{*}(\omega)\right| \\
{\left[\begin{array}{ll}\mathrm{kPa} & \mathrm{s}\end{array}\right]}\end{array}$ & $\begin{array}{l}\text { allest } \omega \\
\text { De }\end{array}$ & $\begin{array}{c}\eta_{0} \text { from } \\
\int_{0}^{\infty} G(t) d t \\
{\left[\begin{array}{ll}\mathrm{kPa} & \mathrm{s}\end{array}\right]}\end{array}$ \\
\hline PS-84k linear & 1.0 & 111.30 & 0.03 & 110.56 & 0.1 & 112.07 \\
\hline PS-185k linear & 12.8 & 1200 & 0.26 & 1170 & 0.86 & 1180 \\
\hline PS-84k ring & 0.040 & 5.71 & 0.07 & 5.76 & 0.04 & 5.61 \\
\hline PS-185k ring & 0.60 & 21.20 & 0.04 & 21.16 & 0.01 & 21.41 \\
\hline
\end{tabular}


Table S2: Experimental steady-state viscosity values for polystyrene ring and linear polymer melts. Reference temperature $T_{\text {ref }}=150^{\circ} \mathrm{C}$.

\begin{tabular}{|c|c|c|c|c|c|c|c|}
\hline \multicolumn{2}{|c|}{$\begin{array}{c}\text { PS-84k } \\
\text { linear }\end{array}$} & \multicolumn{2}{|c|}{$\begin{array}{c}\text { PS-185k } \\
\text { linear }\end{array}$} & \multicolumn{2}{|c|}{$\begin{array}{l}\text { PS-84k } \\
\text { ring }\end{array}$} & \multicolumn{2}{|c|}{$\begin{array}{l}\text { PS-185k } \\
\text { ring }\end{array}$} \\
\hline $\begin{array}{c}\dot{\gamma} \\
{\left[\mathrm{s}^{-1}\right]}\end{array}$ & $\begin{array}{c}\eta \\
{[\mathrm{kPa} s]}\end{array}$ & $\begin{array}{c}\dot{\gamma} \\
{\left[\mathrm{s}^{-1}\right]}\end{array}$ & $\begin{array}{c}\eta \\
{[\mathrm{kPa} s]}\end{array}$ & $\begin{array}{c}\dot{\gamma} \\
{\left[\mathrm{s}^{-1}\right]}\end{array}$ & $\begin{array}{c}\eta \\
{[\mathrm{kPa}}\end{array}$ & $\begin{array}{c}\dot{\gamma} \\
{\left[\mathrm{s}^{-1}\right]}\end{array}$ & $\begin{array}{c}\eta \\
{[\mathrm{kPa} s]}\end{array}$ \\
\hline 0.03 & 111.30 & 0.02 & 1200.00 & 1.75 & 5.71 & 0.06 & 21.20 \\
\hline 0.06 & 112.61 & 0.04 & 1070.00 & 3.12 & 5.39 & 0.10 & 22.30 \\
\hline 0.10 & 112.64 & 0.07 & 879.00 & 5.55 & 5.04 & 0.18 & 20.10 \\
\hline 0.18 & 104.43 & 0.13 & 628.00 & 9.88 & 4.35 & 0.31 & 20.40 \\
\hline 0.32 & 97.25 & 0.23 & 414.00 & 17.54 & 3.67 & 0.56 & 19.40 \\
\hline 0.56 & 90.53 & 0.04 & 259.00 & 31.19 & 2.87 & 0.99 & 16.70 \\
\hline 1.00 & 73.70 & 0.73 & 153.00 & 55.50 & 2.31 & 1.76 & 14.40 \\
\hline 1.78 & 53.20 & 1.29 & 89.30 & 98.79 & 1.68 & 3.12 & 12.10 \\
\hline 3.16 & 35.75 & 2.30 & 50.20 & 175.38 & 1.15 & 5.56 & 9.86 \\
\hline 5.62 & 23.30 & 4.09 & 28.30 & & & 9.89 & 7.73 \\
\hline 10.00 & 14.54 & 7.27 & 16.50 & & & 17.56 & 5.84 \\
\hline 17.80 & 9.31 & & & & & 31.22 & 4.27 \\
\hline 31.60 & 6.40 & & & & & 55.56 & 3.04 \\
\hline 56.20 & 3.83 & & & & & 98.89 & 1.80 \\
\hline
\end{tabular}

\section{Simulation Results}

In Table S3, we list the results of equilibrium molecular dynamics runs where by observing fluctuations and using proper fluctuation-dissipation relations we found terminal relaxation times and zero shear viscosities of all our samples. Table S4 shows results from the MD simulations for steady state viscosity $\eta$ and the eigenvalues $\lambda_{1}, \lambda_{2}$, and $\lambda_{3}$ of the gyration tensor for linear polymers in a melt, for several chain lengths. Table S5 shows similar results for the melts of unconcatenated rings.

In Figure S2, we present results for the eigenvalues of gyration tensor for rings. Each of these eigenvalues, $\lambda_{i}$, depends on the shear rate $\dot{\gamma}$; we plot in Figure S2 ratios $\lambda_{i}(\dot{\gamma}) / \lambda_{i}^{\text {eq }}$, where equilibrium values $\left.\lambda_{i}^{\mathrm{eq}} \equiv \lambda_{i}(\dot{\gamma})\right|_{\dot{\gamma}=0}$. 
Table S3: Terminal relaxation time and zero shear rate viscosity determined from equilibrium simulations by observing equilibrium fluctuations and employing Green-Kubo fluctuationdissipation relations. This data was used to normalize both vertical and horizontal axes to transform Figure 6 in Figure 7.

\begin{tabular}{|c|c|c|c|}
\hline \multicolumn{2}{|c|}{} & $\tau_{\text {relax }} / \tau_{\text {LJ }}$ & $\eta_{0} \sigma^{3} / \varepsilon \tau$ \\
\hline \multirow{4}{*}{ linear } & $N=100$ & $1.59 \cdot 10^{4}$ & $4.13 \cdot 10^{2}$ \\
\cline { 2 - 4 } & $N=200$ & $3.61 \cdot 10^{5}$ & $3.28 \cdot 10^{3}$ \\
\cline { 2 - 4 } & $N=400$ & $1.89 \cdot 10^{6}$ & $3.39 \cdot 10^{4}$ \\
\cline { 2 - 4 } & $N=800$ & $1.69 \cdot 10^{7}$ & $4.86 \cdot 10^{5}$ \\
\hline \hline \multirow{4}{*}{$\operatorname{ring}$} & $N=100$ & $3.35 \cdot 10^{3}$ & $6.06 \cdot 10^{1}$ \\
\cline { 2 - 4 } & $N=200$ & $3.51 \cdot 10^{4}$ & $2.22 \cdot 10^{2}$ \\
\cline { 2 - 4 } & $N=400$ & $1.13 \cdot 10^{5}$ & $4.94 \cdot 10^{2}$ \\
\cline { 2 - 4 } & $N=800$ & $7.89 \cdot 10^{5}$ & $1.68 \cdot 10^{3}$ \\
\hline
\end{tabular}
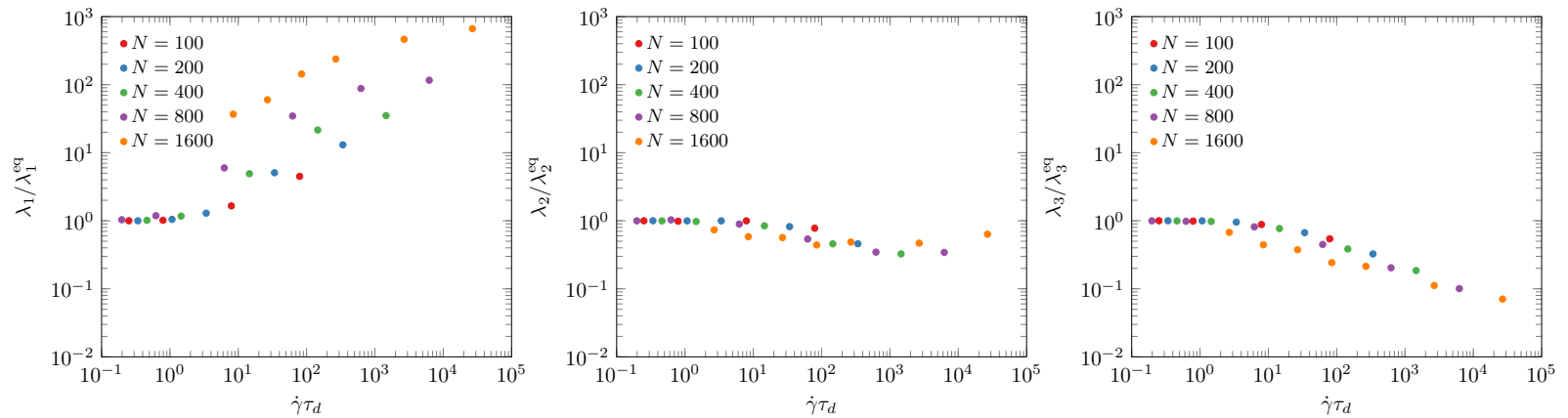

Figure S2: Eigenvalues $\lambda_{1}, \lambda_{2}$, and $\lambda_{3}\left(\lambda_{1}>\lambda_{2}>\lambda_{3}\right)$ of the time-averaged gyration tensor for the rings as a function of shear rate $\dot{\gamma}$. The results are normalized by the corresponding eigenvalue for the equilibrium system (at $\dot{\gamma}=0$ ). Numerical values of $\lambda_{i}$ at different values of $\dot{\gamma}$, including $\dot{\gamma}=0$, are listed in Table S5. 
Table S4: Steady state viscosity $\eta$ and eigenvalues $\lambda_{1}, \lambda_{2}$, and $\lambda_{3}$ of the gyration tensor for linear polymers in a melt. $\eta=\eta_{0}$ for $\dot{\gamma}=0$.

\begin{tabular}{|c|c|c|c|c|}
\hline \multicolumn{5}{|c|}{$N=100$} \\
\hline$\dot{\gamma} \tau$ & $\eta \sigma^{3} / \varepsilon \tau_{\mathrm{LJ}}$ & $\lambda_{1} / \sigma^{2}$ & $\lambda_{2} / \sigma^{2}$ & $\lambda_{3} / \sigma^{2}$ \\
\hline 0 & 350 & 33.5 & 7.3 & 2.6 \\
\hline $10^{-5}$ & 400 & 33.7 & 7.2 & 2.5 \\
\hline $10^{-4}$ & 187 & 49.3 & 7.0 & 2.3 \\
\hline $10^{-3}$ & 38.2 & 110.6 & 5.7 & 1.7 \\
\hline $10^{-2}$ & 9.4 & 204 & 3.4 & 1.0 \\
\hline \multicolumn{5}{|c|}{$N=200$} \\
\hline$\dot{\gamma} \tau$ & $\eta \sigma^{3} / \varepsilon \tau_{\mathrm{LJ}}$ & $\lambda_{1} / \sigma^{2}$ & $\lambda_{2} / \sigma^{2}$ & $\lambda_{3} / \sigma^{2}$ \\
\hline 0 & 3280 & 68.3 & 15.2 & 5.4 \\
\hline $10^{-6}$ & 2530 & 69.8 & 14.9 & 5.3 \\
\hline $10^{-5}$ & 1180 & 110.3 & 14.5 & 5.0 \\
\hline $10^{-4}$ & 178 & 239 & 11.7 & 3.7 \\
\hline $10^{-3}$ & 29.2 & 538 & 7.2 & 2.3 \\
\hline $10^{-2}$ & 7.5 & 895 & 4.2 & 1.1 \\
\hline \multicolumn{5}{|c|}{$N=400$} \\
\hline$\dot{\gamma} \tau$ & $\eta \sigma^{3} / \varepsilon \tau_{\mathrm{LJ}}$ & $\lambda_{1} / \sigma^{2}$ & $\lambda_{2} / \sigma^{2}$ & $\lambda_{3} / \sigma^{2}$ \\
\hline 0 & 32500 & 138.1 & 31.4 & 11.2 \\
\hline $10^{-6}$ & 12200 & 266 & 29.4 & 9.5 \\
\hline $10^{-5}$ & 1140 & 631 & 24.5 & 7.2 \\
\hline $10^{-4}$ & 127 & 1241 & 14.5 & 4.7 \\
\hline $10^{-3}$ & 22.5 & 2550 & 8.7 & 2.7 \\
\hline $10^{-2}$ & 6.2 & 3762 & 5.4 & 1.3 \\
\hline \multicolumn{5}{|c|}{$N=800$} \\
\hline$\dot{\gamma} \tau$ & $\eta \sigma^{3} / \varepsilon \tau_{\mathrm{LJ}}$ & $\lambda_{1} / \sigma^{2}$ & $\lambda_{2} / \sigma^{2}$ & $\lambda_{3} / \sigma^{2}$ \\
\hline 0 & 212000 & - & - & - \\
\hline $10^{-7}$ & 103000 & 575.8 & 64.1 & 18.1 \\
\hline $10^{-6}$ & 9080 & 1632.5 & 54.9 & 15.2 \\
\hline $10^{-5}$ & 881 & 2217 & 52.8 & 13.3 \\
\hline $10^{-4}$ & 89 & 5986 & 19.9 & 5.4 \\
\hline $10^{-3}$ & 17 & 10748 & 12.6 & 2.8 \\
\hline
\end{tabular}


Table S5: Steady state viscosity $\eta$ and eigenvalues $\lambda_{1}, \lambda_{2}$, and $\lambda_{3}$ of the gyration tensor for unconcatenated ring polymers in a melt. $\eta=\eta_{0}$ for $\dot{\gamma}=0$.

\begin{tabular}{|c|c|c|c|c|}
\hline \multicolumn{5}{|c|}{$N=100$} \\
\hline$\dot{\gamma} \tau$ & $\eta \sigma^{3} / \varepsilon \tau_{\mathrm{LJ}}$ & $\lambda_{1} / \sigma^{2}$ & $\lambda_{2} / \sigma^{2}$ & $\lambda_{3} / \sigma^{2}$ \\
\hline 0 & 60.4 & 11.4 & 4.08 & 1.8 \\
\hline $10^{-4.5}$ & 60 & 11.4 & 4.1 & 1.8 \\
\hline $10^{-4}$ & 56.1 & 11.5 & 4.0 & 1.8 \\
\hline $10^{-3}$ & 31.6 & 18.8 & 4.1 & 1.6 \\
\hline $10^{-2}$ & 10.1 & 51.0 & 3.2 & 1.0 \\
\hline \multicolumn{5}{|c|}{$N=200$} \\
\hline$\dot{\gamma} \tau$ & $\eta \sigma^{3} / \varepsilon \tau_{\mathrm{LJ}}$ & $\lambda_{1} / \sigma^{2}$ & $\lambda_{2} / \sigma^{2}$ & $\lambda_{3} / \sigma^{2}$ \\
\hline 0 & 225 & 20.0 & 7.5 & 3.4 \\
\hline $10^{-5}$ & 189 & 20.0 & 7.5 & 3.4 \\
\hline $10^{-4}$ & 96.4 & 25.8 & 7.4 & 3.2 \\
\hline $10^{-3}$ & 30.5 & 101.4 & 6.1 & 2.3 \\
\hline $10^{-2}$ & 8.0 & 260.9 & 3.4 & 1.1 \\
\hline \multicolumn{5}{|c|}{$N=400$} \\
\hline$\dot{\gamma} \tau$ & $\eta \sigma^{3} / \varepsilon \tau_{\mathrm{LJ}}$ & $\lambda_{1} / \sigma^{2}$ & $\lambda_{2} / \sigma^{2}$ & $\lambda_{3} / \sigma^{2}$ \\
\hline 0 & 494 & 33.8 & 12.9 & 6.2 \\
\hline $10^{-6}$ & 400 & 34.1 & 12.7 & 6.0 \\
\hline $10^{-5}$ & 315 & 39.6 & 12.6 & 6.0 \\
\hline $10^{-4}$ & 103 & 210.7 & 10.9 & 4.6 \\
\hline $10^{-3}$ & 23.0 & 729.2 & 5.9 & 2.4 \\
\hline $10^{-2}$ & 6.4 & 1187.3 & 4.2 & 1.1 \\
\hline \multicolumn{5}{|c|}{$N=800$} \\
\hline$\dot{\gamma} \tau$ & $\eta \sigma^{3} / \varepsilon \tau_{\mathrm{LJ}}$ & $\lambda_{1} / \sigma^{2}$ & $\lambda_{2} / \sigma^{2}$ & $\lambda_{3} / \sigma^{2}$ \\
\hline 0 & 2000 & 55.9 & 21.1 & 10.6 \\
\hline $10^{-6}$ & 1590 & 66.4 & 21.8 & 10.4 \\
\hline $10^{-5}$ & 405 & 563.0 & 17.1 & 7.8 \\
\hline $10^{-4}$ & 79 & 2550 & 10.5 & 4.2 \\
\hline $10^{-3}$ & 17.4 & 4915.2 & 7.3 & 2.2 \\
\hline $10^{-2}$ & 6.1 & 6501 & 7.2 & 1.1 \\
\hline \multicolumn{5}{|c|}{$N=1600$} \\
\hline$\dot{\gamma} \tau$ & $\eta \sigma^{3} / \varepsilon \tau_{\mathrm{LJ}}$ & $\lambda_{1} / \sigma^{2}$ & $\lambda_{2} / \sigma^{2}$ & $\lambda_{3} / \sigma^{2}$ \\
\hline 0 & 5700 & 51.2 & 48.9 & 44.3 \\
\hline $10^{-6}$ & 1868 & 727.1 & 35.9 & 29.9 \\
\hline $10^{-5}$ & 310 & 3069.5 & 27.7 & 16.6 \\
\hline $10^{-4}$ & 65.0 & 12166 & 23.8 & 9.46 \\
\hline $10^{-3}$ & 16.0 & 23748 & 22.9 & 5.0 \\
\hline $10^{-2}$ & 5.9 & 34009 & 31.0 & 3.1 \\
\hline
\end{tabular}




\section{Shear Slit Model}

\section{3a. Analytical expressions and limits}

To supplement the information in the main text, the analytical scaling expressions for all scaling regimes are listed in Tables $\mathrm{S} 6$ for the case $N>N_{e}^{3 / 2}$. The corresponding relevant plots are given in Figure 10 in the main text. Table S7 and corresponding Figure S3 are for $N<N_{e}^{3 / 2}$

Table S6: Scaling relations for both shear and tension blobs, as well as chain overall extension in flow $x$-direction $L_{x} \sim\left(N / 2 g_{t}\right) \xi_{t}$ for $N>N_{e}^{3 / 2}$. Corresponding plots are given in Figure 10 in the main text.

\begin{tabular}{|c|c|c|c|c|c|}
\hline Range & $g_{t}$ & $g_{s}$ & $\xi_{t} / b$ & $\xi_{s} / b$ & $L_{x} / b$ \\
\hline $\begin{array}{c}g_{s}=g_{t}=N \\
\dot{\gamma} \tau_{e} \simeq \frac{\tau_{e}}{\tau_{\text {relax }}} \simeq\left(\frac{N_{e}}{N}\right)^{7 / 3}\end{array}$ & $N$ & $N$ & $N_{e}^{1 / 6} N^{1 / 3}$ & $N_{e}^{1 / 6} N^{1 / 3}$ & $N_{e}^{1 / 6} N^{1 / 3}$ \\
\hline $\begin{array}{c}N>g_{s}>g_{t}>N_{e} \\
\left(\frac{N_{e}}{N}\right)^{7 / 3}<\dot{\gamma} \tau_{e}<\left(\frac{N_{e}}{N}\right)^{7 / 4}\end{array}$ & $\frac{N_{e}^{4}}{\left(\dot{\gamma} \tau_{e}\right)^{12 / 7} N^{3}}$ & $\frac{N_{e}}{\left(\dot{\gamma} \tau_{e}\right)^{3 / 7}}$ & $\frac{N_{e}^{3 / 2}}{\left(\dot{\gamma} \tau_{e}\right)^{4 / 7} N}$ & $\frac{N_{e}^{1 / 2}}{\left(\dot{\gamma} \tau_{e}\right)^{1 / 7}}$ & $\frac{\left(\dot{\gamma} \tau_{e}\right)^{8 / 7} N^{3}}{N_{e}^{5 / 2}}$ \\
\hline $\begin{array}{c}N>g_{s}>N_{e}>g_{t}>1 \\
\left(\frac{N_{e}}{N}\right)^{7 / 4}<\dot{\gamma} \tau_{e}<\left(\frac{N_{e}^{3 / 2}}{N}\right)^{7 / 4}\end{array}$ & $\frac{N_{e}^{3}}{\left(\dot{\gamma} \tau_{e}\right)^{8 / 7} N^{2}}$ & $\frac{N_{e}}{\left(\dot{\gamma} \tau_{e}\right)^{3 / 7}}$ & $\frac{N_{e}^{3 / 2}}{\left(\dot{\gamma} \tau_{e}\right)^{4 / 7} N}$ & $\frac{N_{e}^{1 / 2}}{\left(\dot{\gamma} \tau_{e}\right)^{1 / 7}}$ & $\frac{\left(\dot{\gamma} \tau_{e}\right)^{4 / 7} N^{2}}{N_{e}^{3 / 2}}$ \\
\hline $\begin{array}{c}N>g_{s}>N_{e}>g_{t}=1 \\
\left(\frac{N_{e}^{3 / 2}}{N}\right)^{7 / 4}<\dot{\gamma} \tau_{e}<1\end{array}$ & 1 & $\frac{N_{e}}{\left(\dot{\gamma} \tau_{e}\right)^{3 / 7}}$ & 1 & $\frac{N_{e}^{1 / 2}}{\left(\dot{\gamma} \tau_{e}\right)^{1 / 7}}$ & $N$ \\
\hline $\begin{array}{c}N_{e}>g_{s}>g_{t}=1 \\
1<\dot{\gamma} \tau_{e}<N_{e}^{2}\end{array}$ & 1 & $\frac{N_{e}}{\left(\dot{\gamma} \tau_{e}\right)^{1 / 2}}$ & 1 & $\frac{N_{e}^{1 / 2}}{\left(\dot{\gamma} \tau_{e}\right)^{1 / 4}}$ & $N$ \\
\hline
\end{tabular}

\section{3b. Interpolation expressions for relaxation modulus $G(t)$}

\section{Rings}

For simulated rings, the convenient interpolation formula for $G(t)$ was derived in the work ref $^{9}$ (see equation (A.2) from the Supporting Material in that paper). Here, we present this very formula in slightly different notations:

$$
G(t)=G^{\star} \frac{\left[\left(t / \tau^{\star}\right)^{-0.75}+\left(t / \tau^{\star}\right)^{-0.42}\right]}{2} \exp \left[-t / \tau_{\text {relax }}\right]
$$

where $\tau_{\text {relax }}$ was simultaneously fitted for all simulated $N(100,200,400,800,1600)$ according 
Table S7: Scaling relations for both shear and tension blobs, as well as chain overall extension in flow $x$-direction $L_{x} \sim\left(N / 2 g_{t}\right) \xi_{t}$ for $N<N_{e}^{3 / 2}$. Corresponding plots are given in Figure S3.

\begin{tabular}{|c|c|c|c|c|c|}
\hline Range & $g_{t}$ & $g_{s}$ & $\xi_{t} / b$ & $\xi_{s} / b$ & $L_{x} / b$ \\
\hline $\begin{array}{c}g_{s}=g_{t}=N \\
\dot{\gamma} \tau_{e} \simeq \frac{\tau_{e}}{\tau_{\text {relax }}} \simeq\left(\frac{N_{e}}{N}\right)^{7 / 3}\end{array}$ & $N$ & $N$ & $N_{e}^{1 / 6} N^{1 / 3}$ & $N_{e}^{1 / 6} N^{1 / 3}$ & $N_{e}^{1 / 6} N^{1 / 3}$ \\
\hline $\begin{array}{c}N>g_{s}>g_{t}>N_{e} \\
\left(\frac{N_{e}}{N}\right)^{7 / 3}<\dot{\gamma} \tau_{e}<\left(\frac{N_{e}}{N}\right)^{7 / 4}\end{array}$ & $\frac{N_{e}^{4}}{\left(\dot{\gamma} \tau_{e}\right)^{12 / 7} N^{3}}$ & $\frac{N_{e}}{\left(\dot{\gamma} \tau_{e}\right)^{3 / 7}}$ & $\frac{N_{e}^{3 / 2}}{\left(\dot{\gamma} \tau_{e}\right)^{4 / 7} N}$ & $\frac{N_{e}^{1 / 2}}{\left(\dot{\gamma} \tau_{e}\right)^{1 / 7}}$ & $\frac{\left(\dot{\gamma} \tau_{e}\right)^{8 / 7} N^{3}}{N_{e}^{5 / 2}}$ \\
\hline $\begin{array}{c}N>g_{s}>N_{e}>g_{t}>1 \\
\left(\frac{N_{e}}{N}\right)^{7 / 4}<\dot{\gamma} \tau_{e}<1\end{array}$ & $\frac{N_{e}^{3}}{\left(\dot{\gamma} \tau_{e}\right)^{8 / 7} N^{2}}$ & $\frac{N_{e}}{\left(\dot{\gamma} \tau_{e}\right)^{3 / 7}}$ & $\frac{N_{e}^{3 / 2}}{\left(\dot{\gamma} \tau_{e}\right)^{4 / 7} N}$ & $\frac{N_{e}^{1 / 2}}{\left(\dot{\gamma} \tau_{e}\right)^{1 / 7}}$ & $\frac{\left(\dot{\gamma} \tau_{e}\right)^{4 / 7} N^{2}}{N_{e}^{3 / 2}}$ \\
\hline $\begin{array}{c}N>N_{e}>g_{s}>g_{t}>1 \\
1<\dot{\gamma} \tau_{e}<\left(\frac{N_{e}^{3 / 2}}{N}\right)^{4 / 3}\end{array}$ & $\frac{N_{e}^{3}}{\left(\dot{\gamma} \tau_{e}\right)^{3 / 2} N^{2}}$ & $\frac{N_{e}}{\left(\dot{\gamma} \tau_{e}\right)^{1 / 2}}$ & $\frac{N_{e}^{3 / 2}}{\left(\dot{\gamma} \tau_{e}\right)^{3 / 4} N}$ & $\frac{N_{e}^{1 / 2}}{\left(\dot{\gamma} \tau_{e}\right)^{1 / 4}}$ & $\frac{\left(\dot{\gamma} \tau_{e}\right)^{3 / 4} N^{2}}{N_{e}^{3 / 2}}$ \\
\hline $\begin{array}{c}N>N_{e}>g_{s}>g_{t}=1 \\
\left(\frac{N_{e}^{3 / 2}}{N}\right)^{4 / 3}<\dot{\gamma} \tau_{e}\end{array}$ & 1 & $\frac{N_{e}}{\left(\dot{\gamma} \tau_{e}\right)^{1 / 2}}$ & 1 & $\frac{N_{e}^{1 / 2}}{\left(\dot{\gamma} \tau_{e}\right)^{1 / 4}}$ & $N$ \\
\hline
\end{tabular}
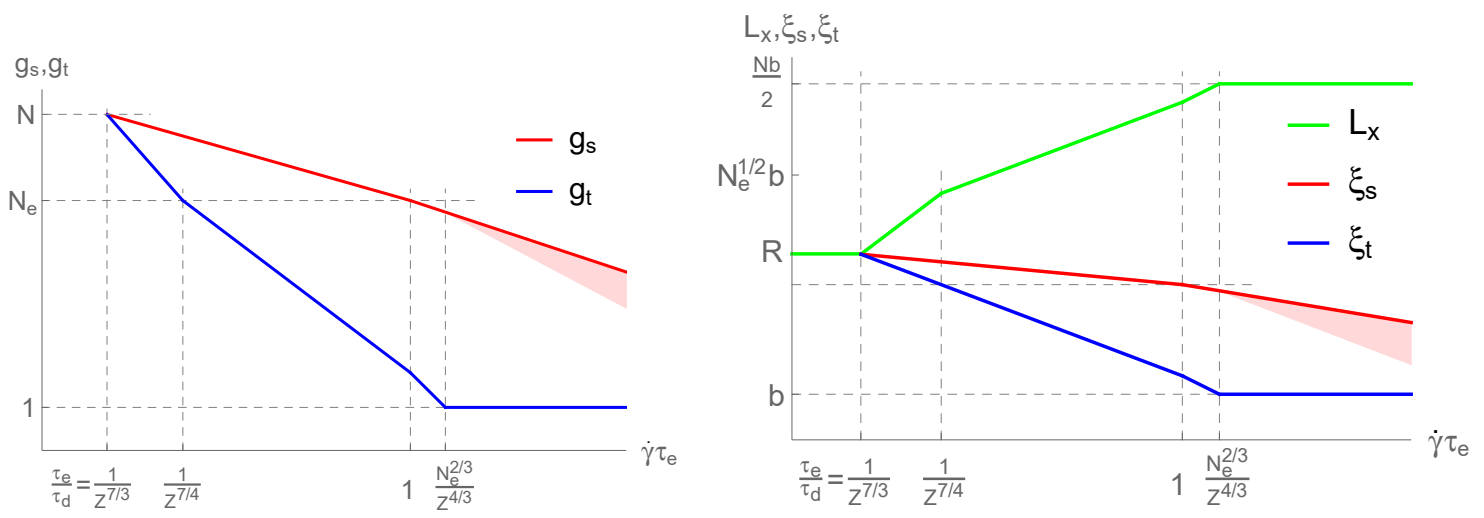

Figure S3: Shear rate dependencies on double logarithmic scale of shear and tension blobs lengths $g_{s}$ and $g_{t}$ and sizes $\xi_{s}$ and $\xi_{t}$, as well as polymer size along the flow $x$ direction, $L_{x} \simeq\left(N / g_{t}\right) \xi_{t}$ for $N<N_{e}^{3 / 2}$. If tension blob becomes as short as one Kuhn segment, further increase of shear rate leads to Kuhn segments increasingly orienting along the flow and reducing the shear blob size. Since we do not analyze this process in details, we show this region as a colored wedge of increasing width. 
to

$$
\tau_{\text {relax }}=\tau_{e}\left(N / N_{e}\right)^{2.40}
$$

Note that $2.40 \approx 7 / 3$ and $0.42 \approx 3 / 7$, while $N_{e}=28$ for simulations in this work. In these equations, $G^{\star}, \tau^{\star}$, and $\tau_{e}$ served as fitting parameters, and the following values were obtained (see ref. ${ }^{9}$ and Supplementary Information of that paper):

$$
\tau^{\star}=8.73 \tau_{\mathrm{LJ}}, \quad G^{\star}=0.25 \frac{\varepsilon}{\sigma^{3}}, \quad \text { and } \tau_{e}=238 \tau_{\mathrm{LJ}}
$$

In the expression (S1a), the 3/4 power in the first term describes undulation modes of semi-flexible chains. In the context of simulations, this is justified because we used the model with $U(\theta)=k_{\theta} \cos \theta$ bond bending potential, with coefficient $k_{\theta}=1.5 \varepsilon$, increasing Kuhn length to $2.8 \sigma$ (from $1.8 \sigma$ for flexible chains with $k_{\theta}=0$ ). We used this model, because entanglement length for fully flexible chains is as long as about 68 beads, leading to many Kuhn segments between entanglements. This restricts simulation on modern computers to relatively small values of $N / N_{e}=Z$. Thus, stiffness was increased in order to decrease entanglement length to $N_{e} \approx 28$, thereby significantly reducing the number of Kuhn monomers per entanglement. This allowed simulation of much higher values of $Z=N / N_{e}$, but significantly reduced the window of Rouse modes in the transition zone, while increasing the window of undulation modes of semi-flexible chains. In fact, this is not at all unphysical, because real modes in experiments are neither pure Rouse nor pure undulations, they are somewhat intermediate and system-dependent. Accordingly, we used formula (S1a) to fit both simulations and experiments data, with the understanding that time $\tau^{\star}$ is an effective value accounting for a complex crossover regime from undulation modes via Rouse modes of a flexible chain (which don't have room to fully develop) to the fractal globule modes.

We used the same interpolation formula (S1a) to fit also experimental data on rings, 
Table S8: Best fit parameters for formula (S1a) for both simulations and experiments data for rings. Fit of simulations data is copied from the work. ${ }^{9}$

\begin{tabular}{|c|c|c|c|}
\hline \multicolumn{4}{|c|}{ Simulation } \\
\hline & $\begin{array}{c}G^{\star} \\
{\left[\varepsilon / \sigma^{3}\right]}\end{array}$ & $\begin{array}{c}\tau^{\star} \\
{\left[\tau_{\mathrm{LJ}}\right]}\end{array}$ & $\begin{array}{c}\tau_{\text {relax }} \\
{\left[\tau_{\mathrm{LJ}}\right]}\end{array}$ \\
\hline Rings & 0.25 & 8.73 & $\begin{array}{c}238\left(N / N_{e}\right)^{2.40} \\
N_{e}=28\end{array}$ \\
\hline \multicolumn{3}{|c|}{ Experiment } \\
\hline sample & $G^{\star}$ & $\tau^{\star}$ & $\tau_{\text {relax }}$ \\
& {$[\mathrm{kPa}]$} & {$[\mathrm{s}]$} & {$[\mathrm{s}]$} \\
\hline Rings 84k & 300 & 0.0015 & 0.12 \\
\hline Rings 185k & 302 & 0.0017 & 1.19 \\
\hline
\end{tabular}

except we considered $G^{\star}, \tau^{\star}$, and $\tau_{\text {relax }}$ is fitting parameters. Best fit parameters are listed in the Table S8, and corresponding plots in Figure S5 demonstrate good agreement with data.

\section{Linear chains}

For linear chains, we use interpolation formula

$$
G(t)=G^{\star}\left[\frac{\left(t / \tau^{\star}\right)^{-0.6 c}+1}{2}\right]^{1 / c} e^{-t / \tau_{\text {relax }}}
$$

with $G^{\star}, \tau^{\star}, c$, and $\tau_{\text {relax }}$ as fit parameters. The motivation behind this formula is as follows. The first power law term in the square brackets, with exponent -0.6 , represents the transition zone; the constant second term in square brackets represents rubbery plateau; power $c$ controls the crossover between the two; and the exponential outside the bracket represents the terminal relaxation mode. This simple fitting formula ignores tube length fluctuations, constraint release modes, and other relaxation modes besides the main one (as the objective is to obtain the terminal time and the viscosity controlled by the product of terminal time and plateau modulus).

The values of best fit parameters for all samples examined in this work, both in simulations and in experiments, are listed in Table S9. Figure S4 shows reasonable fits of the simulation data to this simple model, while Figure S5 shows a similar quality fit for experimental data. 
In particular, the value of $c$ for experimental linear PS84k sample was difficult to fit accurately, because of limited amount of data at small times (high frequencies). Since $c$ was close to unity, the curves for linear chains in Figure S5 are shown for $c=1$.

Table S9: Best fit parameters for formula (S2) for both simulations and experiments data for linear chains.

\begin{tabular}{|c|c|c|c|c|}
\hline \multicolumn{6}{|c|}{ Simulation } \\
\hline$N$ & $\begin{array}{c}G^{\star} \\
{\left[\varepsilon / \sigma^{3}\right]}\end{array}$ & $\begin{array}{c}\tau^{\star} \\
{\left[\tau_{\mathrm{LJ}}\right]}\end{array}$ & $c$ & $\begin{array}{c}\tau_{\text {relax }} \\
{\left[\tau_{\mathrm{LJ}}\right]}\end{array}$ \\
\hline Linear 100 & 0.0415 & 472 & 1 & $1.53 \cdot 10^{4}$ \\
Linear 200 & 0.030 & 1300 & 0.47 & $1.25 \cdot 10^{5}$ \\
Linear 400 & 0.055 & 413 & 0.52 & $1.90 \cdot 10^{6}$ \\
Linear 800 & 0.075 & 204 & 0.72 & $1.70 \cdot 10^{7}$ \\
\hline \multicolumn{5}{|c|}{ Experiment } \\
\hline sample & $G^{\star}$ & $\tau^{\star}$ & $c$ & $\tau_{\text {relax }}$ \\
{$[\mathrm{kPa}]$} & {$[\mathrm{s}]$} & & {$[\mathrm{S}]$} \\
\hline Linear 84k & 215 & 0.01 & $\approx 1$ & 1.0 \\
\hline Linear 185k & 306 & 0.01 & $\approx 1$ & 12 \\
\hline
\end{tabular}

Here, we need to comment on the difference between the values of $\tau_{\text {relax }}$ for $185 \mathrm{k}$ linear chains indicated in Tables S1 (12.8s) and S9 (12s): there is no logical contradiction. One method was based on $G(\omega)$ measurements, led to $12.8 \mathrm{~s}$, and was used to find Wi in Figure 5 and subsequent in the main text, while another method to fit $G(t)$ led to $12 \mathrm{~s}$ and was used to check CM rule. Of course, this difference indicates the numerical inaccuracies involved.

\section{The limit of infinite rings}

We now use interpolation formula (S1a) along with (S1b, S1c) to address the limit of $\tilde{\eta} / \eta_{0}$ when $N \rightarrow \infty$, i.e., the quantity

$$
\frac{\tilde{\eta}(\dot{\gamma})}{\eta_{0}}=\frac{\int_{0}^{1 / \dot{\gamma}} G(t) d t}{\int_{0}^{\infty} G(t) d t}
$$




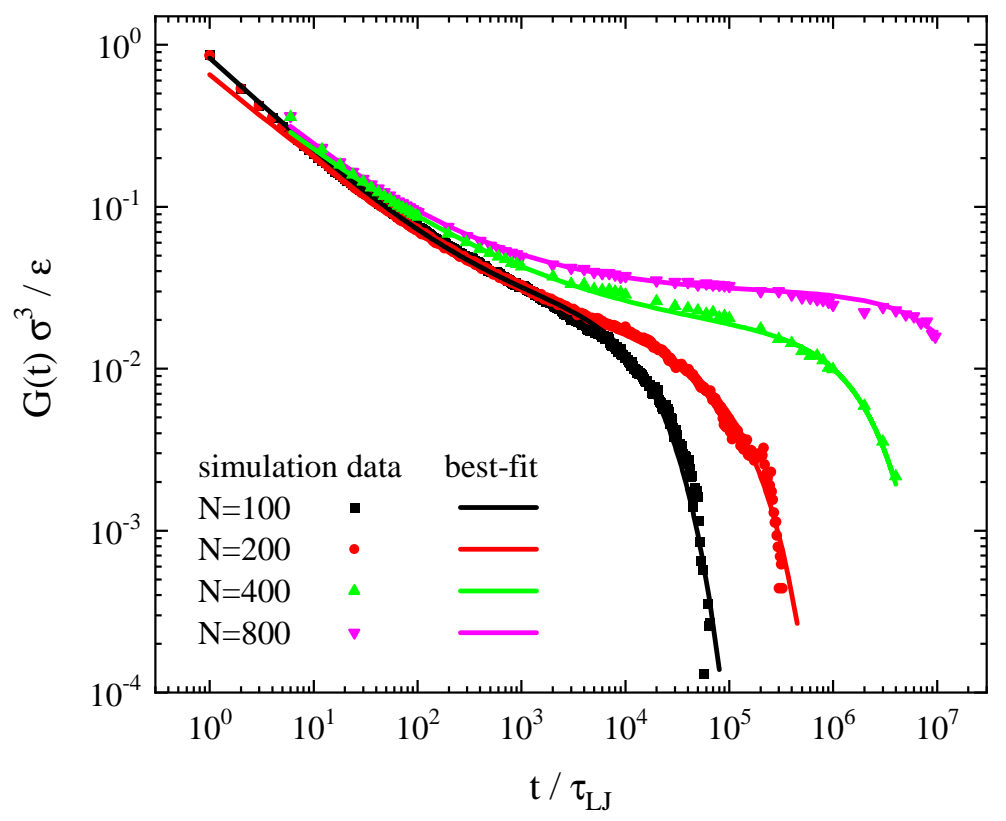

Figure S4: Simulations data for stress relaxation modulus $G(t)$ for linear chains fitted to formula (S2), with best fit parameters listed in the Table S9.

We define the new variable $u=t / \tau_{\text {relax }}$ and then obtain

$$
\begin{aligned}
\frac{\tilde{\eta}(\dot{\gamma})}{\eta_{0}} & =\frac{\int_{0}^{1 / \mathrm{Wi}}\left[\left(u \cdot \frac{\tau_{\text {relax }}}{\tau^{\star}}\right)^{-0.75}+\left(u \cdot \frac{\tau_{\text {relax }}}{\tau^{\star}}\right)^{-0.42}\right] e^{-u} d u}{\int_{0}^{\infty}\left[\left(u \cdot \frac{\tau_{\text {relax }}}{\tau^{\star}}\right)^{-0.75}+\left(u \cdot \frac{\tau_{\text {relax }}}{\tau^{\star}}\right)^{-0.42}\right] e^{-u} d u} \\
& =\frac{\int_{0}^{1 / \mathrm{Wi}} u^{-0.75} e^{-u} d u+\left(\frac{\tau^{\star}}{\tau_{\text {relax }}}\right)^{0.33} \int_{0}^{1 / \mathrm{Wi}} u^{-0.42} e^{-u} d u}{\int_{0}^{\infty} u^{-0.75} e^{-u} d u+\left(\frac{\tau^{\star}}{\tau_{\text {relax }}}\right)^{0.33} \int_{0}^{\infty} u^{-0.42} e^{-u} d u} \\
& =\frac{\int_{0}^{1 / \mathrm{Wi}} u^{-0.75} e^{-u} d u+\left(\frac{\tau^{\star}}{\tau_{e}}\right)^{0.33}\left(\frac{N}{N_{e}}\right)^{0.8} \int_{0}^{1 / \mathrm{Wi}} u^{-0.42} e^{-u} d u}{\int_{0}^{\infty} u^{-0.75} e^{-u} d u+\left(\frac{\tau^{\star}}{\tau_{e}}\right)^{0.33}\left(\frac{N}{N_{e}}\right)^{0.8} \int_{0}^{\infty} u^{-0.42} e^{-u} d u},
\end{aligned}
$$

where of course $\mathrm{Wi}=\dot{\gamma} \tau_{\text {relax }}$. In the limit of $N \rightarrow \infty$ all that remains is the second term in both numerator and denominator. As expected, $N$ remains only inside Wi:

$$
\left.\frac{\tilde{\eta}(\dot{\gamma})}{\eta_{0}}\right|_{N \rightarrow \infty}=\frac{\int_{0}^{1 / \mathrm{Wi}} u^{-0.42} e^{-u} d u}{\int_{0}^{\infty} u^{-0.42} e^{-u} d u}=1-\frac{\Gamma(0.58,1 / \mathrm{Wi})}{\Gamma(0.58)},
$$




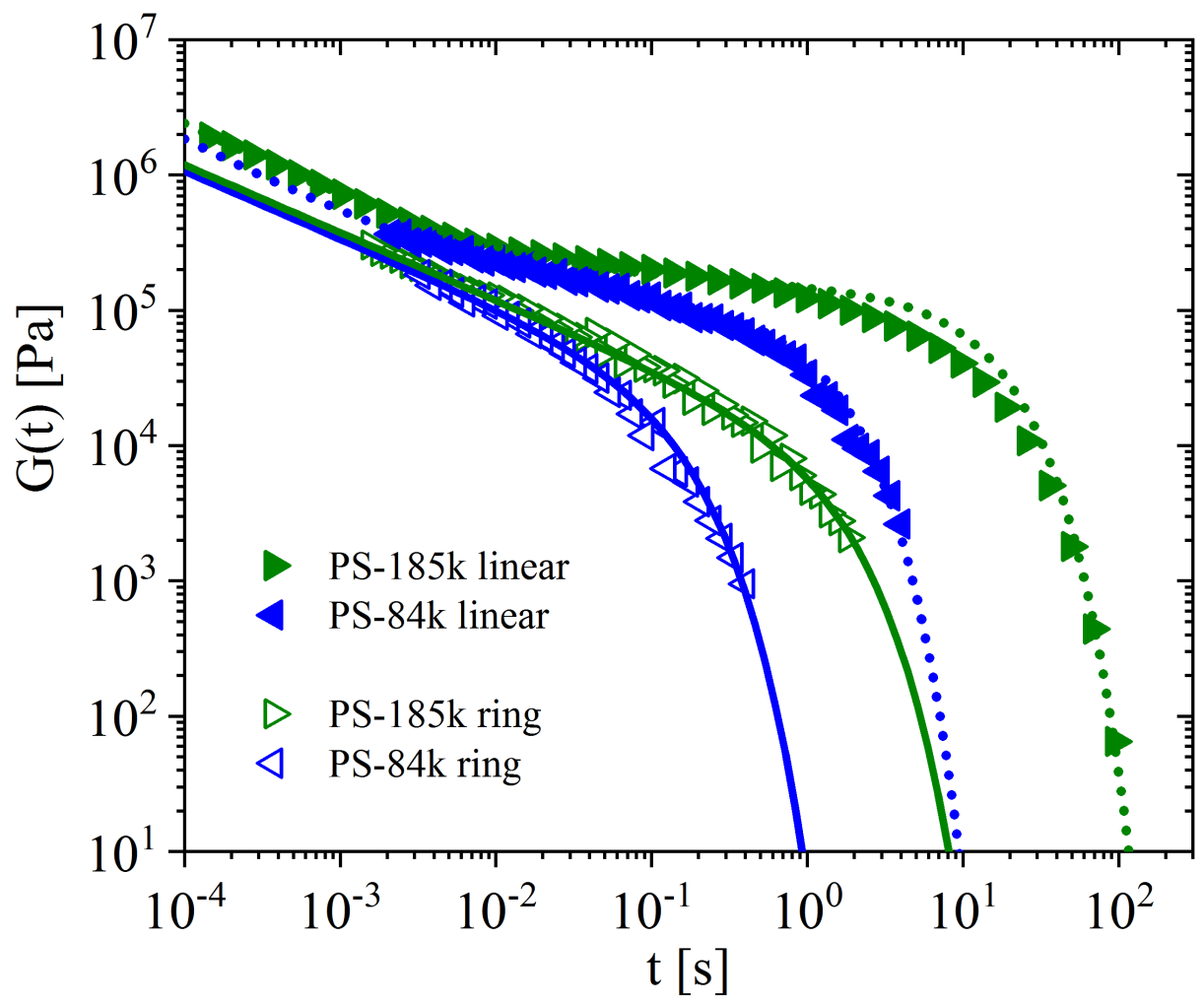

Figure S5: Experimental data for stress relaxation modulus $G(t)$ for rings and linear chains fitted to equations (S1a) and (S2), respectively. Best fit parameters used in these plots are listed in the Tables S8 for rings and S9 for linear chains 
where $\Gamma$ with two arguments is the so-called incomplete $\Gamma$-function, and $\Gamma(0.58) \approx 1.53$. This is plotted as a thick orange line in Figure 9 in the main text, in double logarithmic plot it has the slope of $-0.58 \approx-4 / 7$.

\section{Cox-Merz rule}

\section{4a. Two forms of Cox-Merz rule}

We now return to the Cox-Merz rule, discussed repeatedly in the main text. Despite its success and usefulness, its applicability has not been fully justified yet. The plots of Figures 5 and 7 indicate, for both experiments and simulations, that steady-shear viscosity $\eta(\dot{\gamma})$ is closely approximated by the absolute value of complex viscosity $\left|\eta^{*}(\omega)\right|$ for both linear and ring entangled polymers at modest $\mathrm{Wi}$, validating this rule.

We formulated a new form of Cox-Merz rule using the quantity $\tilde{\eta}(\omega)=\int_{0}^{1 / \omega} G(t) d t$ instead of $\left|\eta^{*}(\omega)\right|$. Its main advantage is its physical justification based on our naive scaling theory. Figure 9 verifies the rule in this new form at least for rings. The traditional form of Cox-Merz rule is difficult to justify from the theoretical standpoint, because the use absolute value in $\left|\eta^{*}(\omega)\right|$ has no physical significance, especially given that it involves both storage and loss moduli. Nevertheless, this traditional form of the rule is convenient in the context of experiments, because complex viscosity $\eta^{*}(\omega)$ is directly measured.

Here we analyze more systematically these two forms of Cox-Merz rule and compare them.

\section{4b. Two forms of Cox-Merz rule and experiments}

In Figure S6, we show experimental data for steady shear viscosity $\eta(\dot{\gamma})$ for both linear polymers and rings along with both absolute value of complex viscosity $\left|\eta^{*}(\omega)\right|$ and the $\tilde{\eta}(\omega)$ value (the latter is defined in formula (6) in the main text: $\left.\tilde{\eta}(\omega)=\int_{0}^{1 / \omega} G(t) d t\right)$.

To make our point, we followed a somewhat non-direct route. We took measured values of dynamic moduli $G^{\prime}(\omega)$ and $G^{\prime \prime}(\omega)$, performed numerical Fourier transform to find stress 


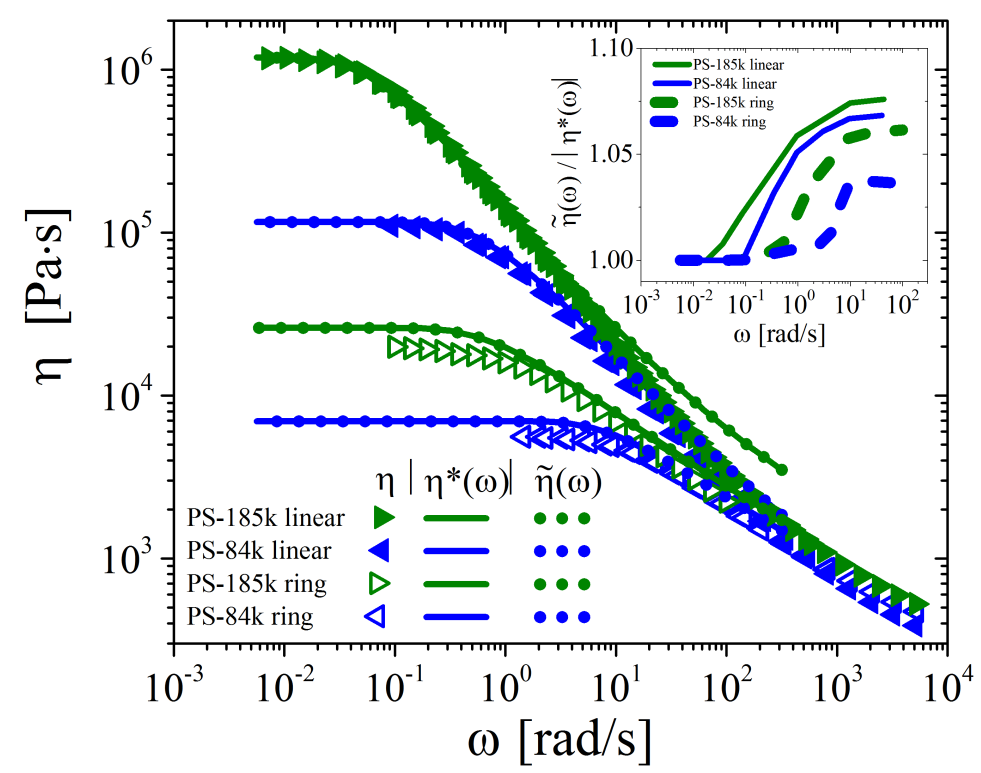

Figure S6: Experimental data for steady shear viscosity $\eta$ as a function of shear rate $\dot{\gamma}$ along with absolute value of complex viscosity $\left|\eta^{*}(\omega)\right|$ and the incomplete integral $\tilde{\eta}(\omega)=$ $\int_{0}^{1 / \omega} G(t) d t$, both as functions of frequency $\omega$, and both computed based on a fitted analytical expressions for stress relaxation modulus $G(t)$, formulas (S1a) and (S2). Ratio $\tilde{\eta}(\omega) /\left|\eta^{*}(\omega)\right|$ is plotted in the inset. Both forms of Cox-Merz rule work about equally well.

relaxation modulus $G(t)$, then fitted it to an analytical formula as described above, Eq. (S1a), and then used this analytical formula to find numerically both $\left|\eta^{*}(\omega)\right|$ and $\tilde{\eta}(\omega)$. The reason why we do it like this is because our goal here is rather limited, we only want to compare two forms of Cox-Merz rule. The limited accuracy of measurements at hight frequency $\omega$ and/or at high shear rate $\dot{\gamma}$ is an important, but entirely different question.

Overall, Figure S6 suggests that both forms of Cox-Merz rule are about equally accurate and break down in about the same way with increasing $\mathrm{Wi}$ in excess of 10 or so. The use of one of them or the other is, therefore, in this sense, entirely a matter of practical convenience. Nevertheless, we emphasize that our new form of Cox-Merz rule is derived and justified based on physics, while the traditional form is purely empirical.

\section{4c. Two forms of Cox-Merz rule and simulations}

Figure S7 shows two forms of Cox-Merz rule against simulations data for linear chains (A) and for rings (B). To generate this figure, we followed the same principle as in case of 
experiments, i.e., we used stress relaxation modulus data to find an analytical fit of $G(t)$, and then used it to numerically compute both $\left|\eta^{*}(\omega)\right|$ and $\tilde{\eta}(\omega)$. The results are also similar to those in case of experiments, with both forms of Cox-Merz rule working equally well at modest Wi and breaking down in about the same way at larger Wi.
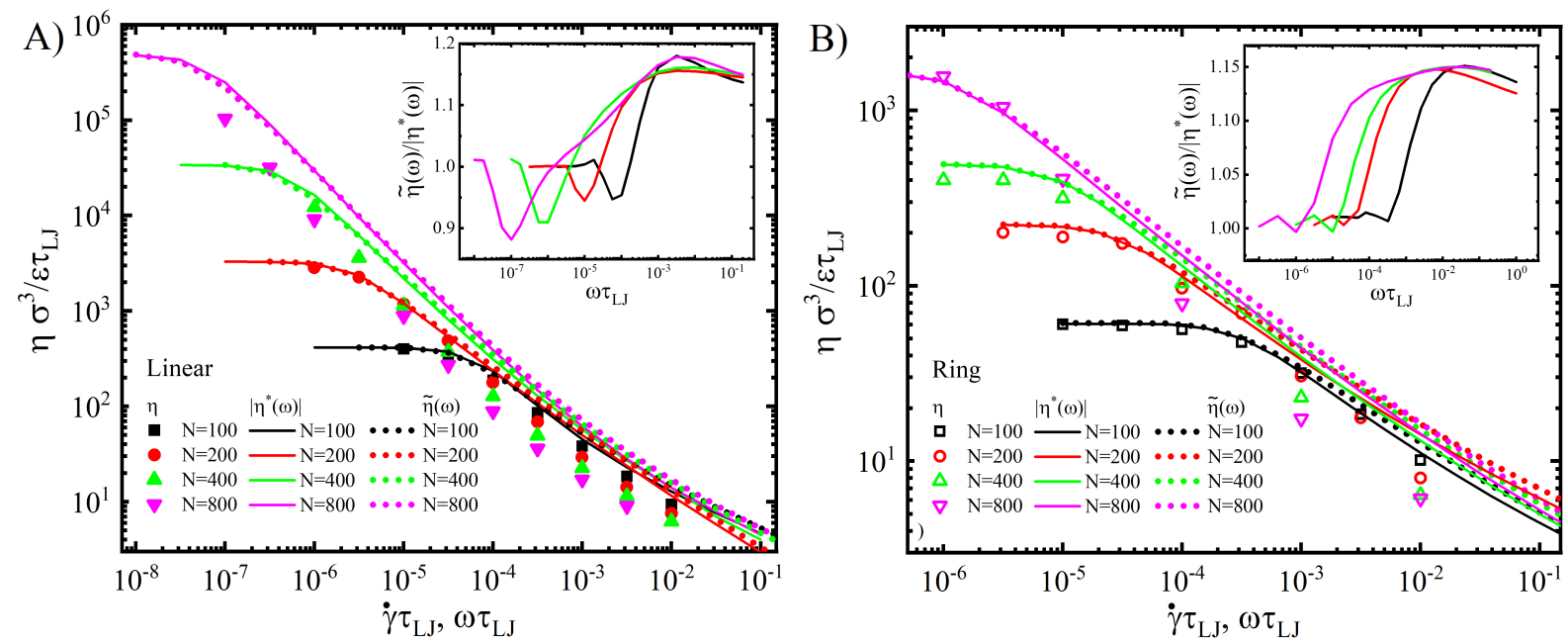

Figure S7: Simulations data for steady shear viscosity $\eta$ as a function of shear rate $\dot{\gamma}$ along with absolute value of complex viscosity $\left|\eta^{*}(\omega)\right|$ and the incomplete integral $\tilde{\eta}(\omega)=$ $\int_{0}^{1 / \omega} G(t) d t$ as functions of frequency $\omega$. Panel A for linear chains and panel B for rings. Both $\tilde{\eta}(\omega)$ and $\left|\eta^{*}(\omega)\right|$ are computed based on a fitted analytical expression for stress relaxation modulus $G(t)$, formulas (S2) and (S1a) for linear chains and rings, respectively. Ratio $\tilde{\eta}(\omega) /\left|\eta^{*}(\omega)\right|$ is plotted in the inset. Both forms of Cox-Merz rule work about equally well.

\section{4d. Mathematics of two forms of Cox-Merz rule}

Cox-Merz rule is traditionally formulated in terms of absolute value of complex viscosity $\left|\eta^{*}(\omega)\right|=\left|\int_{0}^{\infty} G(t) e^{i \omega t} d t\right|$, while we suggested (Eq. 6) to use instead $\tilde{\eta}(\omega)=\int_{0}^{1 / \dot{\gamma}} G(t) d t$. We show below that for any reasonable function $G(t)$, both of these quantities are within about $15 \%$ of one another, meaning that either both form of the rule work or both don't, but they are, roughly, equivalent. To show this, we consider the purely mathematical question of the numerical value of the ratio

$$
\frac{\tilde{\eta}(\omega)}{\left|\eta^{*}(\omega)\right|}=\frac{\int_{0}^{1 / \omega} G(t) d t}{\left[\left(\int_{0}^{\infty} \sin \omega t G(t) d t\right)^{2}+\left(\int_{0}^{\infty} \cos \omega t G(t) d t\right)^{2}\right]^{1 / 2}}
$$


for various realistic functions $G(t)$. We show below that this ratio is usually within about $15 \%$ of unity.

First, this ratio is exactly one in the limit $\omega \rightarrow 0$. In the opposite limit $\omega \rightarrow \infty$, it is not one, but close. For instance, if $G(t)=t^{-\delta} e^{-t}$ (for brevity, in this section we measure $t$ in units of $\left.\tau_{\text {relax }}\right)$, then this limit equals $1 / \Gamma(2-\delta)$, which is 1 at $\beta=0$ and at $\beta=1$ and reaches its maximum of 1.14 at $\delta \approx 0.58$ (plotted in the inset of Figure S8 as a function of $\delta)$. Figure S8 presents numerically computed ratio (S6) for a few typical $G(t)$ and illustrates the idea that $\int_{0}^{1 / \dot{\gamma}} G(t) d t$ and $\left|\eta^{*}(\omega)\right|$ are numerically pretty close to one another. The same conclusion can be drawn also from the insets of Figures S6 and S7 where this same ratio is plotted for several other $G(t)$ functions. A note of caution: we are not claiming that the ratio of integrals (S6) is close to unity for any mathematically possible function $G(t)$. For instance, if $G(t)=t^{-\delta} e^{-t}$, then this ration can become very small for the unphysical $\delta<0$, as seen in the inset in Figure S8.

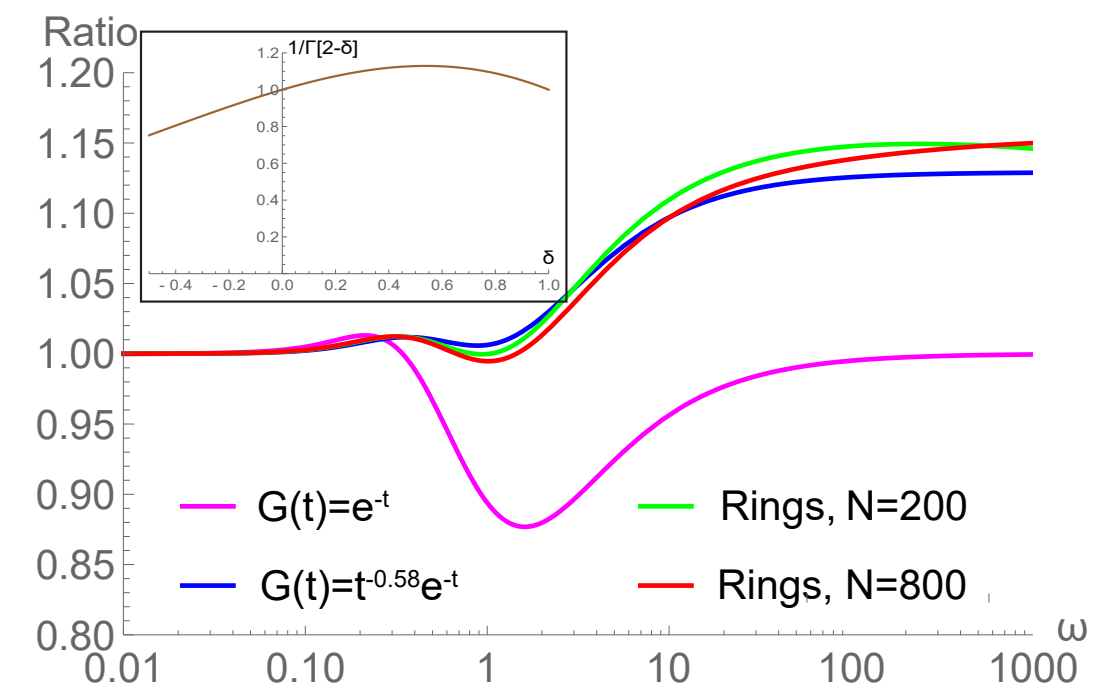

Figure S8: Ratio of viscosities defined as an incomplete integral of relaxation modulus and as absolute value of complex viscosity, Eq. (S6), for a variety of $G(t)$ functions. In this plot, $\tau_{\text {relax }}$ is taken to be the unit of time, which means that $\omega$ is both Wi and De. The inset shows the value of this ratio in $\omega \rightarrow \infty$ limit for $G(t)=t^{-\delta} e^{-t}$ as a function of $\delta$. 


\section{Ring Stretched along a Line}

As we have seen in the main text, ring in nonlinear shear regime stretch significantly along the flow direction $x$. Here, we address an auxiliary problem analyzing both of our geometric characteristics of conformations, eigenvalues of the gyration tensor and end-to-end distances of chain sections, specifically for the strongly stretched ring.

First let us imagine a ring of $N$ monomers maximally stretched to the length $L=N \sigma / 2$. In this case, gyration eigenvalue is well known (see e.g. ref. $^{2}$ )

$$
\lambda_{1}=L^{2} / 12
$$

Finding the end-to-end distance of chain section with $k$ bonds, between monomers $m$ and $n=m+k$, is a bit more subtle. Of course, for a strongly stretched linear chain the answer would be trivial: $k \sigma$. For a ring, there are at least two kinks in an extended ring and if one of these kinks happens to be inside the $k$-segment, the distance would be reduced (with small probability this projection could be even close to zero if the kink is at the center of the $k$-segment). The exact results for averaged distance and mean squared distance are (assuming for simplicity $N$ is even)

$$
\begin{aligned}
& \frac{1}{\sigma}\left\langle\left|x_{k}\right|\right\rangle=\left[1-\frac{2 k}{N}\right] k+\sum_{q=(N / 2)-k}^{(N / 2)-1}\left[\frac{2}{N}\right]|N-k-2 q|, \\
& \frac{1}{\sigma^{2}}\left\langle x_{k}^{2}\right\rangle=\left[1-\frac{2 k}{N}\right] k^{2}+\sum_{q=(N / 2)-k}^{(N / 2)-1}\left[\frac{2}{N}\right](N-k-2 q)^{2},
\end{aligned}
$$

where the first and the second terms in both equations correspond to the cases of absent or present kink inside $k$-section, respectively; expressions in the square brackets are probabili- 
ties. If $N$ is large, we can replace sums by integrals, yielding

$$
\begin{aligned}
& \frac{1}{\sigma}\left\langle\left|x_{k}\right|\right\rangle=k-k^{2} / N, \\
& \frac{1}{\sigma^{2}}\left\langle x_{k}^{2}\right\rangle=k^{2}-4 k^{3} / 3 N .
\end{aligned}
$$

This is plotted in figure S9.
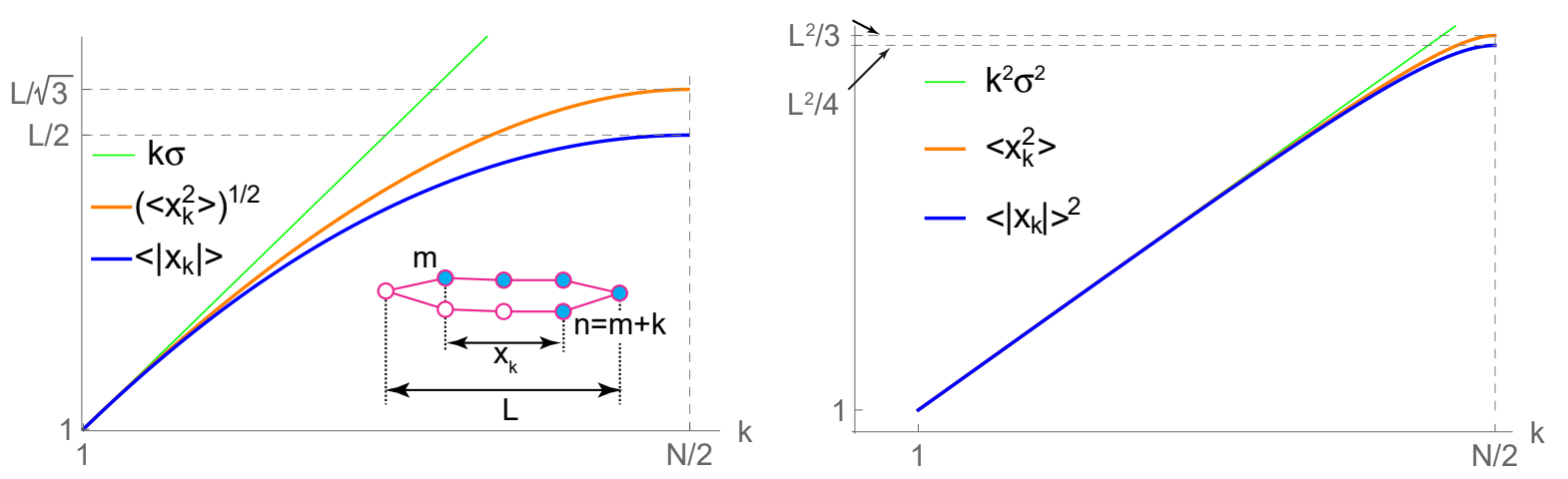

Figure S9: For a ring strongly stretched along $x$, as shown in the inset, mean and rootmean-squared distance between monomers $m$ and $n=m+k$ in linear scale is shown in the left figure, while squared average and averaged squared distances are shown in double logarithmic scale in the right figure. In all cases averages are taken over $m$. For a larger $k$-section, the distance is smaller than $k$ because of the increasing chance that one of the inevitable U-turns happens to be inside the $k$-section.

Of course, $\left\langle x_{k}^{2}\right\rangle$ reaches maximum for $k=N / 2$, and this maximal value is $\left\langle x_{N / 2}^{2}\right\rangle=L^{2} / 3$. In other words, according to Eq. (S7)

$$
\left\langle x_{N / 2}^{2}\right\rangle=4 \lambda_{1}
$$

This relation is significantly more general, valid not only for maximally stretched ring. Indeed, let monomers labelled by $-N / 2<q<N / 2$, and let coordinate of monomer $q$ be $r_{q}$, with $r_{N / 2}=r_{-N / 2}\left(\right.$ ring closure condition) and with $\left|r_{0}-r_{N / 2}\right|=L$, meaning both halves of the ring, $q<0$ and $q>0$, have the same end-to-end distance. Suppose further that ring is 
stretched such that its center of mass is at the middle, $\int_{-N / 2}^{N / 2} r_{q} d q=0$. Then

$$
\begin{aligned}
\lambda_{1} & =\frac{1}{N} \int_{-N / 2}^{N / 2} r_{q}^{2} d q \\
\left\langle x_{N / 2}^{2}\right\rangle & =\frac{2}{N} \int_{0}^{N / 2}\left[r_{q}-r_{q-N / 2}\right]^{2} d q
\end{aligned}
$$

and simple algebra manipulations yield the announced relation $\left\langle x_{N / 2}^{2}\right\rangle=4 \lambda_{1}$ (S10). This is why this relation is so useful in our analysis of stretched conformations in nonlinear shear regime, even though they are of course not completely stretched. 